\title{
Bluer colour of UX Arietis at fainter visual magnitudes
}

\author{
M. J. Rosario ${ }^{1}$, A. V. Raveendran ${ }^{2}$, and M. V. Mekkaden ${ }^{2}$ \\ 1 Vainu Bappu Observatory, Indian Institute of Astrophysics, Kavalur, India \\ e-mail: mjr@iiap.res.in \\ 2 Indian Institute of Astrophysics, Bangalore 560034, India \\ e-mail: [avr;mvm]@iiap.res.in
}

Received 7 August 2007 / Accepted 12 September 2007

\section{ABSTRACT}

\begin{abstract}
Aims. The RS CVn star UX Ari appears bluer when it is fainter, which is unusual for a spotted star; we aim to resolve this unusual behaviour of the object. Also the data available in the literature indicate that the mean $V$ magnitude of UX Ari varies by about 25 years; we want to confirm whether this is indeed true.

Methods. The most important parameter in understanding the colour variation is the relative brightnesses of the component stars of UX Ari. We use all spectroscopic information available in the literature to obtain a reasonable value for this parameter. We also present new $B V$ photometry of UX Ari obtained on 58 nights during 2001-07.

Results. The $V$ magnitudes of UX Ari we obtained are significantly fainter than those reported in the literature, and they are not consistent with a 25 -year period for the mean light level. The increased fractional contribution by the hotter G-type component to the total light in the blue spectral region as the active star becomes faint, seems to be the real cause for the bluer colour of UX Ari at fainter visual magnitudes.
\end{abstract}

Key words. stars: activity - binaries: spectroscopic - stars: individual: UX Ari - stars: late-type - starspots

\section{Introduction}

UX Ari (HD 21242, BD $+28^{\circ} 532$ ) is a bright member of the RS Canum Venaticorum binaries, and is one of the few objects for which extensive $B V$ photometry exists. It has been observed almost every season since the discovery of its light variability in 1972 by Hall et al. (1975) (Ulvas \& Henry 2003, and the references therein). It is a non-eclipsing, double-lined, 6.44 day binary, and its orbital elements were first derived by Carlos \& Popper (1971) who tentatively gave the spectral types G5 V and K0 IV to the components. More recently, its orbital elements have been refined using high resolution, high signal-to-noiseratio spectra by Duemmler \& Aarum (2001) who found that the star is a triple system. The third component apparently does not contribute to the total light of the system. However, the above authors have detected a system of weak lines in the spectra of UX Ari, which they attribute to another star in its line of sight.

From an analysis of the long-term photometry Ulvas \& Henry (2003) find that UX Ari displays a 25-year period in its mean $V$ magnitude.

The light variability seen in RS CVn binaries has been attributed to starspots, analogues to sunspots, present on the active component of the binary system. One would expect the starspots, which are presumed to be cooler than the surrounding photosphere, to make the star redder at fainter visual magnitudes, and in fact most RS CVn systems behave as such. The unusual behaviour of UX Ari was first pointed out by Wacker et al. (1986), who found that the intermediate band colour index, ([4530]-[6585]), is bluer when the star is fainter. Subsequent observations by several investigators confirmed the out-of-phase variation of $(B-V)$ with the $V$ light curve (Wacker \& Guinan 1987; Mohin \& Raveendran 1989; Rodono \& Cutispoto 1992; Raveendran \& Mohin 1995).
Since there was no quantitative estimation of the relative brightnesses of the components, Raveendran \& Mohin (1995) used the qualitative description of the spectra of UX Ari in the photographic and visual regions given by Carlos \& Popper (1971) and argued that the bluer colour of the star at light minimum is due to an increase in the fractional contribution to the total light in the blue spectral region by the hotter star in the system, as suggested by Wacker et al. (1986).

Rodono \& Cutispoto (1992) had put forward an alternative explanation: the anti-correlation between the $V$ and $(B-V)$ variations exhibited by UX Ari is probably caused by high temperature phenomena, such as continuous flaring activity or plage-like activity associated with the spots on the cooler active star. More recently, Ulvas \& Engvold (2003a) developed this idea into a spot model in which the dark spots are assumed to be surrounded by bright faculae. Using this model, they tried to reproduce the observed trend in the $(B-V)$ versus $V$ variation. This model was later extended to account for the colour variations seen in V711 Tau and the prototype RS CVn by Ulvas \& Henry (2005). In the case of the latter star they find that faculae cooler than those needed in the cases of UX Ari and V711 Tau are to be invoked to represent the $(B-V)$ variation more accurately.

We find that the parameters of the components of UX Ari used by Ulvas \& Engvold (2003a) in their modeling are not consistent with the plots of relative brightnesses of the components in the spectral region 5800-8700 $\AA$ (Ulvas \& Engvold 2003b) and the qualitative description of the spectra given by Carlos \& Popper (1971) earlier.

In this paper we present new $B V$ photometry of UX Ari obtained during 2001-07 and discuss the mechanism which causes the out-of-phase variation of $(B-V)$ colour with its $V$ magnitude. 
Table 1. $B V$ photometry of UX Ari.

\begin{tabular}{lrrlrr}
\hline \hline JD & & & JD & & \\
$2450000+$ & $\Delta V$ & $\Delta(B-V)$ & $2450000+$ & $\Delta V$ & $\Delta(B-V)$ \\
\hline 1950.157 & 0.988 & -0.216 & 2688.136 & 1.040 & -0.202 \\
1951.139 & 1.022 & -0.229 & 2691.162 & 1.139 & -0.265 \\
1952.163 & 1.035 & -0.221 & 2692.155 & 1.094 & \\
1953.141 & 1.005 & -0.216 & 2699.086 & 1.052 & -0.243 \\
1958.111 & 1.029 & -0.233 & 3018.120 & 1.146 & -0.279 \\
1971.147 & 1.039 & -0.217 & 3021.118 & 0.972 & -0.231 \\
2252.207 & 1.038 & -0.230 & 3023.099 & 1.031 & -0.201 \\
2253.267 & 1.025 & -0.242 & 3024.138 & 1.085 & -0.239 \\
2254.325 & 1.052 & -0.238 & 3025.182 & 1.175 & -0.270 \\
2256.295 & 1.017 & -0.230 & 3026.102 & 1.177 & -0.291 \\
2290.102 & 1.056 & -0.215 & 3027.154 & 1.020 & -0.200 \\
2292.145 & 1.030 & -0.228 & 3045.114 & 1.179 & -0.275 \\
2293.106 & 1.026 & -0.225 & 3759.159 & 1.246 & -0.259 \\
2321.122 & 1.037 & -0.193 & 3760.176 & 1.160 & -0.258 \\
2328.121 & 1.075 & -0.238 & 3767.150 & 1.114 & -0.249 \\
2329.101 & 1.078 & -0.237 & 3768.123 & 1.058 & -0.211 \\
2330.087 & 1.065 & -0.227 & 3772.109 & 1.230 & -0.256 \\
2341.100 & 1.042 & -0.254 & 3774.152 & 1.074 & \\
2346.114 & 1.010 & -0.248 & 3786.100 & 1.147 & -0.235 \\
2605.294 & 1.092 & -0.227 & 3787.103 & 1.062 & -0.215 \\
2606.315 & 1.122 & -0.229 & 3789.106 & 1.059 & -0.227 \\
2608.307 & 1.102 & -0.228 & 4136.107 & 1.103 & -0.201 \\
2609.330 & 1.016 & -0.232 & 4137.106 & 1.163 & -0.232 \\
2610.277 & 1.018 & -0.225 & 4138.142 & 1.235 & -0.257 \\
2611.254 & 1.069 & -0.225 & 4139.114 & 1.243 & -0.250 \\
2622.242 & 1.017 & -0.233 & 4141.130 & 1.137 & -0.243 \\
2661.144 & 1.017 & -0.233 & 4152.103 & 1.262 & \\
2664.201 & 1.123 & -0.257 & 4154.100 & 1.133 & -0.220 \\
2668.157 & 1.017 & -0.218 & 4173.103 & 1.177 & -0.254 \\
\hline & & & & &
\end{tabular}

\section{New BV photometry}

UX Ari was observed photometrically in $B V$ bands on 58 nights during 2001-07 with the 34-cm telescope of Vainu Bappu Observatory (VBO), Kavalur. All measurements were made differentially with respect to the comparison star 62 Ari (G5 III) and transformed to the $U B V$ system. HR 999 (K2 II-III) was observed on several nights along with the variable and comparison stars for checking the stability of the photometric system and for estimating the errors in the differential magnitudes. Table 1 lists the results of photometric observations. Each value given in the table is a mean of 2-4 independent measurements. The typical uncertainty in both differential $V$ and $(B-V)$ values is $\sim 0.01 \mathrm{mag}$. The differential $V$ magnitudes given in Table 1 are plotted in Fig. 1 along with those compiled by Ulvas \& Henry (2003), which in addition to their own data, include those obtained by Hall et al. (1975), Hall (1977), Landis et al. (1978), Guinan et al. (1981), Sarma \& Prakasa Rao (1984), Mohin \& Raveendran (1989), Strassmeier et al. (1989), Raveendran \& Mohin (1995) and Padmakar \& Pandey (1996).

The previously reported observations indicated a fairly smooth variation for the mean $V$ magnitude of UX Ari with a period around 25 years (Ulvas \& Henry 2003). The present observations, however, are not consistent with such a period. The faintest differential $V$ magnitude $(\Delta V)$ observed earlier during $1984-1985$ by Busso et al. (1986) is around 1.17. The present observations show a $\Delta V$ of 1.26 mag for the object, which is about $0.10 \mathrm{mag}$ fainter than the above value. The observations obtained during 2001-07 indicate that the $\Delta V$ at light curve minimum has monotonically decreased over the years. Observations during the coming seasons will be very important to uncover whether the trend continues further or the object

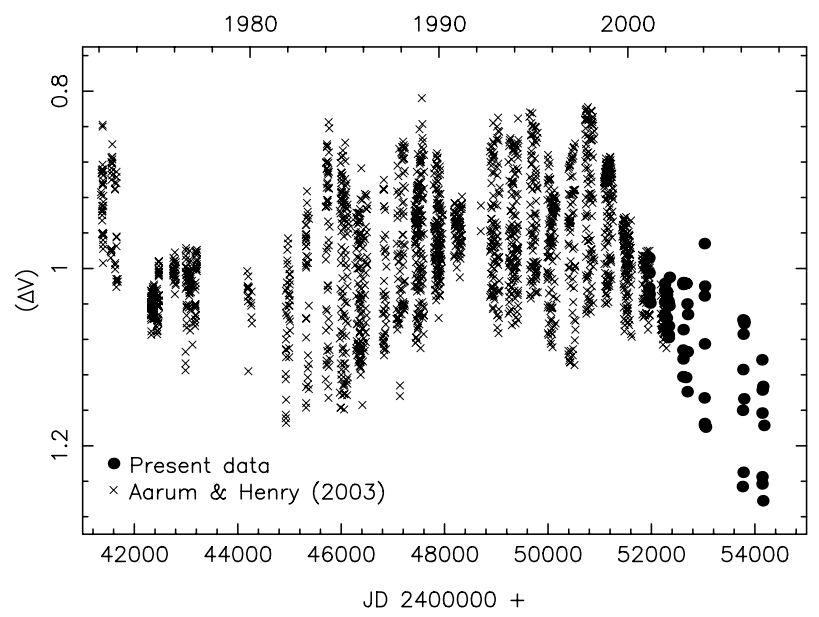

Fig. 1. Plot of $\Delta V$ of UX Ari with respect to 62 Ari against the corresponding Julian day of observation.

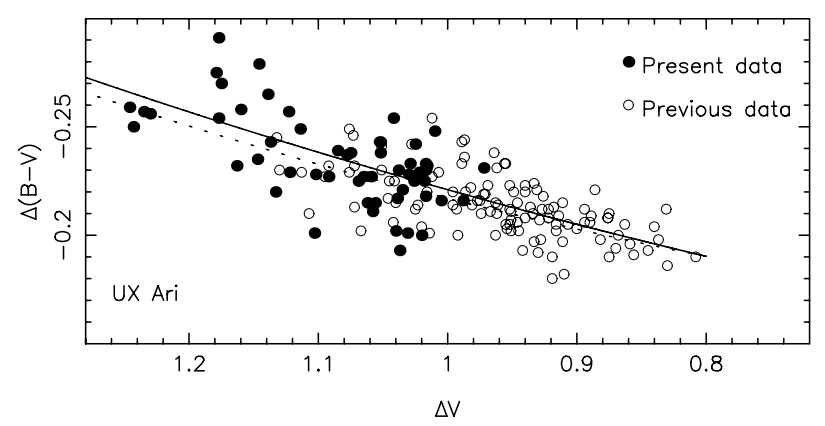

Fig. 2. Plot of $\Delta(B-V)$ of UX Ari with respect to 62 Ari against the corresponding $\Delta V$ obtained at VBO over 1984-2007. The solid and dotted lines represent the expected variation of the composite colour as the brightness of UX Ari varies. In the former case the $(B-V)$ of the active star was assumed to be 1.08 throughout, and in the latter case it was assumed to vary linearly from 1.08 to 1.10 when $\Delta V$ varied from 0.8 to 1.25 .

becomes brighter. The faintest magnitude shown by the star at light curve minimum will give information on the maximum spot coverage that is possible on the stellar surface.

\section{3. $(B-V)$ variation}

The plot of $\Delta(B-V)$ against $\Delta V$ presented by Ulvas \& Engvold (2003a) show a large scatter. In the plot they have included observations by several investigators. Part of the scatter seen in the plot arises from the systematic differences between the different photometric systems employed for the observations. In Fig. 2 we have plotted the $\Delta(B-V)$ against the corresponding $\Delta V$ obtained at VBO over 1984-94 (Mohin \& Raveendran 1989; Raveendran $\&$ Mohin 1995) along with those given in Table 1 . The observations were obtained with the same photomultiplier tube and the same set of filters. The figure clearly shows that UX Ari becomes bluer as it becomes fainter, as reported by several investigators earlier. The $(B-V)$ colour varied by about 0.07 mag when the $V$ magnitude varied by about 0.45 .

In Fig. 3 we have plotted the $\Delta(B-V)$ of DM UMa against the corresponding $\Delta V$ obtained at VBO over 1984-2007, the period covered by the observations of UX Ari. The $(B-V)$ colour of II Peg obtained at VBO during 1984-94 against its $V$ magnitude, also obtained at VBO, is plotted in Fig. 4. All the observations were made with the same photometric setup as that used for the observations of UX Ari. Both DM UMa and II Peg are 


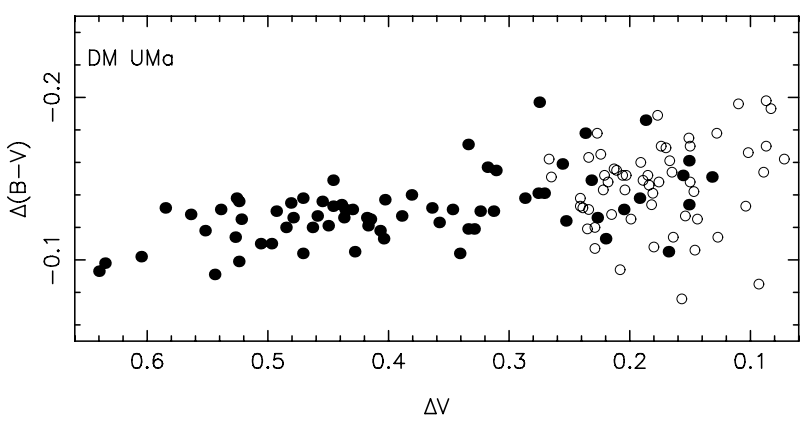

Fig. 3. Plot of $\Delta(B-V)$ of DM UMa with respect to $\mathrm{BD}+60^{\circ} 1301$ against the corresponding $\Delta V$ obtained at VBO over 1984-2007. Filled circles represent data from Mohin \& Raveendran (1992, 1994), and open circles represent unpublished data.

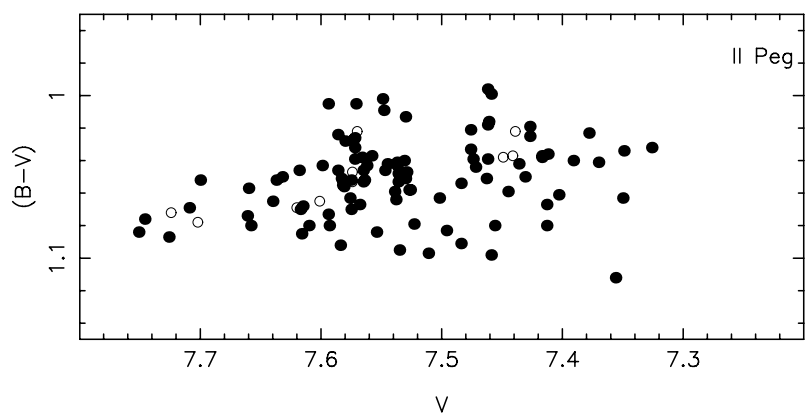

Fig. 4. Plot of $(B-V)$ colour of II Peg against the corresponding $V$ magnitude obtained at VBO over 1984-94. Filled circles represent data from Mohin et al. (1986) and Mohin \& Raveendran (1993), and open circles represent unpublished data.

single-lined binaries, and hence the variations in $(B-V)$ colour with $V$ can be attributed entirely to the active components of the respective binary systems. The visible, active stars of these systems have spectral types K0-K1 III-IV (Kimble et al. 1981) and K2 IV (Vogt 1981), respectively. UX Ari, DM UMa and II Peg along with V711 Tau are the four known RS CVn systems which always show $\mathrm{H} \alpha$ in emission (Wacker et al. 1986), indicating similar magnitudes of spot activity. From Fig. 3 it is quite evident that DM UMa becomes redder as it becomes fainter, consistent with the conventional starspot model. In the case of II Peg, Fig. 4 shows that it is not so evident, but there is definitely an indication that the star behaves similarly.

\section{Relative brightnesses of K- and G-type components}

The observed variations in $V$ or $(B-V)$ cannot be directly attributed to the active K-type star because UX Ari is a doublelined binary. The contribution by the secondary will have to be taken into account while interpreting the variations in $V$ magnitude or $(B-V)$ colour.

Ulvas \& Engvold (2003a) derived the $V$ magnitudes of the G- and unspotted K-type star using the Barnes-Evans relation (Barnes \& Evans 1976) which relates the effective temperature to the unreddened $V$ magnitude and angular size of the star. The $(B-V)$ colours that are typical for the spectral types of the components were then used to calculate their $B$ magnitudes. If we substitute the parameters for the K- and G-type stars, namely, $\left(T_{\text {eff }}\right)_{K}=4750 \mathrm{~K},(\mathrm{BC})_{K}=-0.31,(B-V)_{K}=0.95, R_{K}=5.78 R_{\odot}$, $\left(T_{\mathrm{eff}}\right)_{G}=5620 \mathrm{~K},(\mathrm{BC})_{G}=-0.07,(B-V)_{G}=0.70$ and $R_{G}=1.11 R_{\odot}$, used by Ulvas \& Engvold (2003a), we get the differences in the $V$ and $B$ magnitudes of the G- and unspotted K-type stars as 2.61 and 2.36, respectively. These values are surely inconsistent with the observations of Carlos \& Popper (1971), which showed UX Ari to be a double-lined spectroscopic binary; the spectra of the secondary would have been completely obliterated had it been about 1 mag fainter than the primary (Heintz 1978).

Figure 1 shows that the brightest $\Delta V$ of UX Ari with respect to 62 Ari observed so far is around 0.80. Ulvas \& Engvold (2003a) considered this to represent the magnitude of the system when the K-type star is unspotted. Carlos \& Popper (1971) observed UX Ari spectroscopically for radial velocity measurements during 1967-70. Unfortunately, we do not have photometric measurements of the star during this period. However, Fig. 1 indicates that the mean $V$ magnitude of UX Ari was about 0.15 mag fainter than the brightest magnitude observed. According to the above authors the spectra in the photographic region were dominated by the G-type star with strong absorption lines, and the absorption lines of the K-type star were seen only on closer inspection. However, the spectra in the visual region were dominated by the K-type star. It may be noted here that the Mount Wilson spectral classification (Joy \& Wilson 1949) of UX Ari is dG5, indicating the dominance of the G-type star in the blue spectral region. If we take $(B-V)_{K}=1.0$ and $(B-V)_{G}=0.70$, appropriate for their spectral types, we have $\left(B_{K}-B_{G}\right)+\left(V_{G}-V_{K}\right)=0.30$. Assuming G-type star to dominate in the blue region and K-type star to dominate in the visual region by similar amounts, we have $V_{K}-V_{G}=-0.15 \mathrm{mag}$, when UX Ari is $\sim 0.15$ mag below the maximum observed brightness.

More recently, Ulvas \& Engvold (2003b) derived the brightness of the K-type star relative to that of the G-type star in 6000-8000 $\AA$ region. Their plots show that the difference in $R$ magnitudes in the Kron-Cousins system, in the sense $R_{K}-R_{G}$, lie approximately in the range -0.55 to -0.65 . The $(V-R)$ colours of a main sequence star of $T_{\text {eff }}=5620 \mathrm{~K}$ and a giant star of $T_{\text {eff }}=4750 \mathrm{~K}$ in the above photometric system are 0.37 and 0.54 , respectively (Drilling \& Landolt 2000). These values together with the above difference in $R$ magnitudes show that $V_{K}-V_{G}$ was in the range -0.48 to -0.38 during their observations which span 1995 December-2000 January. Further, if we extrapolate the plots presented by Ulvas \& Engvold (2003b) we find that the relative magnitude of the components at $5500 \AA$ was, most likely, in the range -0.40 to -0.20 during the same period. Figure 1 shows that UX Ari was around 0.15-0.20 mag below the maximum observed brightness around the above period. These orders of estimate indicate that the relative brightnesses of the components are substantially less than what Ulvas \& Engvold (2003a) used in their model, in which they ruled out the increased contribution in the blue by the hotter star and suggested the occurrence of faculae to account for the bluer colour of UX Ari at fainter magnitudes. We think a reasonable value for the difference in $V$ magnitudes of unspotted K- and G-type stars is $\sim 0.6$.

\section{Modelling of $(B-V)$ variation}

From Fig. 2 we find that the brightest and faintest $\Delta V$ values observed so far are 0.80 and 1.25 and the corresponding $\Delta(B-V)$ colours are -0.19 and -0.26 , respectively. The $(B-V)$ of 62 Ari is 1.11 (Hoffleit 1982), and the above differential values indicate that the $(B-V)$ of UX Ari varied between 0.91 and 0.84 when its $V$ magnitude varied by 0.45 . Depending on the difference in the $V$ magnitudes of the components at the maximum 


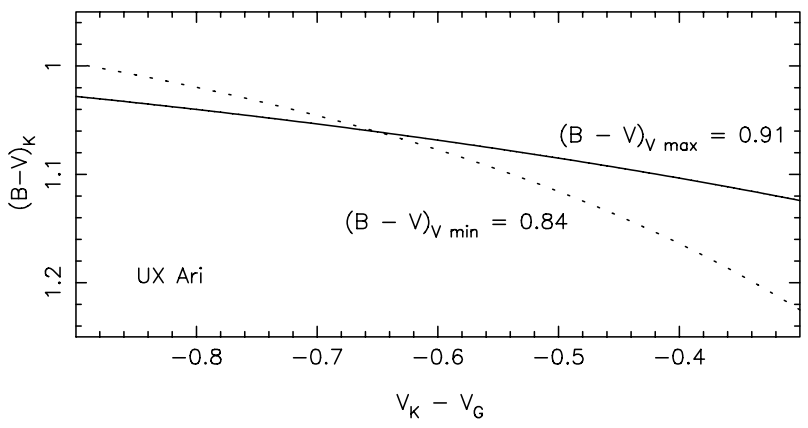

Fig. 5. Plot of the derived $(B-V)$ values of the K-type star at the brightest and faintest observed $V$ magnitudes against the corresponding assumed difference in the unspotted K- and G-type stars. The composite $(B-V)$ colours observed are indicated.

observed brightness, which we take to indicate the case when the active K-type star is unspotted or least spotted, these composite colours would give different $(B-V)$ colours for the K-type star when it is brightest and faintest, if the colour of the G-type star is assumed to be constant.

Taking the $(B-V)$ of the G-type star to be 0.68 (Drilling $\&$ Landolt 2000) we have computed the $(B-V)$ colours of the K-type star which would satisfy the above composite colours for a range of difference between the $V$ mag of the component stars, and the results are presented in Fig. 5. It is clear from the figure that if the magnitude difference is less than about 0.75 then the $\mathrm{K}$-type star would be redder when it is fainter, which is consistent with the conventional spot model and what is observed in the case of single-lined binaries, such as DM UMa and II Peg. For example, if $V_{K}-V_{G}=-0.55$, then the composite colours at the brightest and faintest magnitudes would be satisfied if the $(B-V)$ colours of the K-type star are 1.08 and 1.10, respectively. Taking the above magnitude difference between the components when UX Ari was at the maximum observed brightness, in Fig. 2 we have plotted the expected variation of the composite colour as its $V$ magnitude varied by about 0.45 mag. We made the calculations for the two cases assuming: (i) there is no variation in the $(B-V)$ of the K-type star as its brightness changed and (ii) the $(B-V)$ linearly varied from 1.08 to 1.10 when the $V$ magnitude of UX Ari varied by 0.45 . It is clear from the figure that the computed curves closely match the general trend in the variation of $(B-V)$ with $V$. We, therefore, conclude that UX Ari becomes bluer when it becomes fainter because of the increase in the fractional contribution to the total light in the blue spectral region by the hotter star, and the observations are consistent with the conventional spot model.

All the objects which show or are suspected to show bluer colours at fainter magnitudes, UX Ari, V711 Tau, RS CVn (Ulvas \& Henry 2005) and MM Her (Tas et al. 1999), are doublelined spectroscopic binaries. There is no apparent reason why the spots on the active components of double-lined binaries are different from those on the corresponding components in single-lined binaries. There may be faculae associated with the starspots as in the case of sunspots, however, they are definitely not responsible for the bluer colour of these binaries when they become fainter.

The reason why Ulvas \& Engvold (2003a) had to invoke faculae to account for the bluer colour of UX Ari when it becomes fainter is that they took the ratio of radii $\left(R_{K} / R_{G}\right)$ to be 5.2 in their model and ended up with a rather large flux ratio of $\left(F_{K} / F_{G}\right)=11$. The radii of the components, $5.78 R_{\odot}$ and $1.11 R_{\odot}$ given by Duemmler \& Aarum (2001), which are based on the derived $v \sin i$ values of the components, are not definitely consistent with the spectroscopic observations of Carlos \& Popper (1971) and Ulvas \& Engvold (2003b). Most likely, the $v \sin i$ of the K-type star is over-estimated and that of the G-type star is under-estimated.

\section{Conclusions}

1. New $B V$ photometry obtained over 2001-07 shows $V$ magnitudes which are fainter than those so far reported in the literature. We find that the brightness at light curve minimum decreased monotonically since 2000 . Photometry in the coming next few seasons will be very important to know whether the same trend continues, or the star becomes brighter.

2. The present data do not conform with the earlier observations, which show a period of 25 years for the mean $V$ magnitude of UX Ari.

3. From all the available spectroscopic information on UX Ari we find that the difference in $V$ magnitudes of the G-type star and unspotted or least spotted K-type star is around 0.6, which is substantially smaller than that used by Ulvas \& Engvold (2003a) in their modeling.

4. The bluer colour of UX Ari at fainter $V$ magnitudes can be understood in terms of increased fractional contribution to the total light in the blue spectral region by the hotter G5 star. There is no need to invoke the occurrence of faculae to account for the unusual colour variation observed in UX Ari.

\section{References}

Busso, M., Scaltriti, F., \& Cellino, A. 1986, A\&A, 156, 106

Barnes, T. G., \& Evans, D. S. 1976, MNRAS, 174, 489

Carlos, R. C., \& Popper, D. M. 1971, PASP, 83, 504

Drilling, J. S., \& Landolt, A. U. 2000, in Allen's Astrophysical Quantities, ed. A. N. Cox

Duemmler, R., \& Aarum, V. 2001, A\&A, 370, 974

Guinan, E. F., McCook, G. P., Fragola, J. L., O’Donnell, W. C., \& Weisenberger, A. G. 1981, PASP, 93, 495

Hall, D. S. 1977, Acta Astron., 27, 281

Hall, D. S., Montle, R. E., \& Atkins, H. R. 1975, Acta Astron., 25, 125

Heintz, W. D. 1978, Double stars (Dordrecht, Holland: D. Reidel Publishing Company)

Hoffleit, D. 1982, The Bright Star Catalogue, Yale University Observatory, USA Joy, A. H., \& Wilson, R. E. 1949, ApJ, 109, 231

Kimble, R. A., Kahn, S. M., \& Bowyer, S. 1981, ApJ, 251, 585

Landis, H. L., Lovell, L. P., Hall, D. S., Henry, G. W., \& Renner, T. R. 1978, AJ, 83,176

Mohin, S., \& Raveendran, A. V. 1989, J. Astrophys. Astron., 10, 35

Mohin, S., \& Raveendran, A. V. 1992, A\&A, 256, 487

Mohin, S., \& Raveendran, A. V. 1993, A\&A, 277, 155

Mohin, S., \& Raveendran, A. V. 1994, A\&A, 286, 824

Mohin, S., Raveendran, A. V., \& Mekkaden, M. V. 1986, Bull. Astr. Soc. India, 14,48

Padmakar, S. P., \& Pandey, S. K. 1996, A\&AS, 138, 203

Raveendran, A. V., \& Mohin, S. 1995, A\&A, 301, 788

Rodono, M., \& Cutispoto, G. 1992, A\&AS, 95, 55

Sarma, M. B. K., \& Prakasa Rao, B. V. N. S. 1983, IBVS, 2357

Strassmeier, K. G., Hall, D. S., Boyd, L. J., \& Genet, R. M. 1989, ApJS, 69, 141

Tas, G., Evren, S., \& Ibanoglu 1999, A\&A, 349, 546

Ulvas, V. A., \& Henry, G. W. 2003, A\&A, 402, 1033

Ulvas, V. A., \& Engvold, O. 2003a, A\&A, 399, L11

Ulvas, V. A., \& Engvold, O. 2003b, A\&A, 402, 1043

Ulvas, V. A., \& Henry, G. W. 2005, AN, 326, 292

Vogt, S. S. 1981, ApJ, 247, 975

Wacker, S. W., \& Guinan, E. F. 1987, IBVS, 3018

Wacker, S. W., Guinan, E. F., McCook, G. P., Lochner, J. C., \& Paczkowski, B. G. 1986, IBVS No. 2920 